\title{
Semi-Lagrangian lattice Boltzmann model for compressible flows on unstructured meshes
}

\author{
M. H. Saadat, F. Bösch, and I. V. Karlin $\mathbb{1}^{*}$ \\ Department of Mechanical and Process Engineering, ETH Zurich, 8092 Zurich, Switzerland
}

(Received 16 October 2019; revised manuscript received 21 January 2020; accepted 4 February 2020; published 24 February 2020)

\begin{abstract}
Compressible lattice Boltzmann model on standard lattices [M. H. Saadat, F. Bösch, and I. V. Karlin, Phys. Rev. E 99, 013306 (2019).] is extended to deal with complex flows on unstructured grid. Semi-Lagrangian propagation [A. Krämer et al., Phys. Rev. E 95, 023305 (2017).] is performed on an unstructured second-order accurate finite-element mesh and a consistent wall boundary condition is implemented which makes it possible to simulate compressible flows over complex geometries. The model is validated through simulation of Sod shock tube, subsonic and supersonic flow over NACA0012 airfoil and shock-vortex interaction in Schardin's problem. Numerical results demonstrate that the present model on standard lattices is able to simulate compressible flows involving shock waves on unstructured meshes with good accuracy and without using any artificial dissipation or limiter.
\end{abstract}

DOI: 10.1103/PhysRevE.101.023311

\section{INTRODUCTION}

Lattice Boltzmann method (LBM) $[1,2]$ as a kinetic theory approach to computational fluid dynamics (CFD) is now a well-established tool for simulation of complex fluid flows ranging from turbulence [3,4] and multiphase [5] and multicomponent flows [6] to rarefied gas flows [7], magnetohydrodynamics [8], relativistic hydrodynamics [9], and others. In the LBM, populations $f_{i}(\boldsymbol{x}, t)$ associated with a set of discrete velocities $\mathcal{C}=\left\{\boldsymbol{c}_{i}, i=0, \ldots, Q-1\right\}$ are designed to recover the governing equations of continuum mechanics in the hydrodynamic limit. The evolution of populations is based on simple rules of propagation along the discrete velocities $\mathcal{C}$ and relaxation to a local equilibrium. This makes the LBM a simple and efficient alternative for conventional CFD solvers [10].

Despite these advantages, the most common LB models used in the literature (i.e., standard lattices: the D2Q9 model in two dimensions and D3Q27, D3Q19, and D3Q15 in three dimensions) suffer from a limited Galilean invariance and lack of isotropy at high-speed flows, which make their application limited to low-speed incompressible flows. The number of discrete velocities of the standard lattices is too low to reproduce all the moments required for obtaining the full compressible Navier-Stokes-Fourier (NSF) equations [11]. Increasing the number of discrete velocities and using high-order (multispeed) lattice models is a systematic approach to circumvent these limitations and simulate high-speed compressible flows [12-15]. However, apart from increased computational cost, a

\footnotetext{
*Corresponding author: karlin@lav.mavt.ethz.ch
}

Published by the American Physical Society under the terms of the Creative Commons Attribution 4.0 International license. Further distribution of this work must maintain attribution to the author(s) and the published article's title, journal citation, and DOI. limited temperature range is another restriction of high-order lattices [16]. Several models have also been proposed in the literature based on standard lattices [17-21] for simulation of compressible flows; however, to the best of our knowledge, none of them were so far successful in simulating supersonic flows involving shock waves.

Recently, we proposed an augmented LB model on standard lattices which can recover the full NSF equations with adjustable Prandtl number and adiabatic exponent in the hydrodynamic limit [22]. This was achieved by incorporating appropriate correction terms into the kinetic equations in order to compensate the error terms associated with the low symmetry of the standard lattices. It was shown that the model is isotropic and Galilean invariant. The model was further extended by the concept of the shifted lattices [23] and successfully applied to subsonic and supersonic compressible flows with shocks.

In this paper, we extend the model formulation to unstructured finite-element mesh using a semi-Lagrangian propagation scheme and introduce consistent wall boundary conditions for the simulation of complex geometries. Similarly to the standard LB, the semi-Lagrangian scheme follows the characteristics curve of the LB equation backward in time to find the departure point of each grid node. However, since the propagation is performed on an arbitrary nonuniform grid, interpolation is required to reconstruct the populations at the departure points. Finite-element-based interpolation schemes are good candidates as they allow to have body-conforming meshes which give more flexibility in handling complex geometries and are more efficient in capturing small scale structure of the flow near the wall. Another advantage of the semi-Lagrangian scheme is that the time step can be chosen arbitrarily and it remains stable at large Courant-FriedrichsLewy (CFL) numbers. This is at variance to many other off-lattice schemes (such as finite-difference or finite-volume LB schemes) which operate at restricted CFL number due to explicit time integration, see e.g., Ref. [24]. Note that 
finite-element-based semi-Lagrangian scheme has successfully been applied to incompressible LB models [25,26]. Here we apply the semi-Lagrangian scheme with second-order accurate finite-element interpolation to the compressible LB model [22] to test its capabilities for simulation of compressible flows on unstructured grid.

The outline of the paper is as follows: The augmented LB model is reviewed in Sec. II for the sake of completeness. Detailed numerical implementation of the model on unstructured mesh and the consistent wall boundary conditions are presented in Sec. III. In Sec. IV, the model is validated through simulation of several benchmark test cases. Finally, conclusions are drawn in Sec. V.

\section{MODEL DESCRIPTION}

The kinetic equations of the compressible LB model with variable Prandtl number and adiabatic exponent are as follows [22]:

$$
\begin{aligned}
f_{i}(\boldsymbol{x}, t)-f_{i}\left(\boldsymbol{x}-\boldsymbol{v}_{i} \delta t, t-\delta t\right)= & \omega\left(f_{i}^{\mathrm{eq}}-f_{i}\right)+\delta t \phi_{i}, \\
g_{i}(\boldsymbol{x}, t)-g_{i}\left(\boldsymbol{x}-\boldsymbol{v}_{i} \delta t, t-\delta t\right)= & \omega\left(g_{i}^{\mathrm{eq}}-g_{i}\right) \\
& +\left(\omega_{1}-\omega\right)\left(g_{i}^{*}-g_{i}\right),
\end{aligned}
$$

where $\phi_{i}$ are correction terms responsible for canceling out the spurious terms in the momentum equation, resulting from lack of isotropy of the standard lattices, $g_{i}^{*}$ is a quasiequilibrium population, and $f_{i}^{\mathrm{eq}}, g_{i}^{\mathrm{eq}}$ are local equilibria which satisfy the local conservation laws for the density $\rho$, momentum $\rho \boldsymbol{u}$, and total energy $\rho E$,

$$
\begin{gathered}
\sum_{i=0}^{Q-1}\left\{1, \boldsymbol{v}_{i}\right\} f_{i}=\sum_{i=0}^{Q-1}\left\{1, \boldsymbol{v}_{i}\right\} f_{i}^{\mathrm{eq}}=\{\rho, \rho \boldsymbol{u}\} \\
\sum_{i=0}^{Q-1} g_{i}=\sum_{i=0}^{Q-1} g_{i}^{\mathrm{eq}}=2 \rho E
\end{gathered}
$$

The temperature is defined by

$$
T=\left(1 / C_{v}\right)\left(E-u^{2} / 2\right)
$$

where $C_{v}$ is the specific heat of ideal gas at constant volume. The relaxation parameters $\omega$ and $\omega_{1}$ are related to the dynamic viscosity $\mu$ and thermal conductivity $\kappa$,

$$
\begin{gathered}
\mu=\left(\frac{1}{\omega}-\frac{1}{2}\right) \rho T \delta t, \\
\kappa=C_{p}\left(\frac{1}{\omega_{1}}-\frac{1}{2}\right) \rho T \delta t .
\end{gathered}
$$

Below a system of units is used where the universal gas constant is set to one, $R=1$. Consequently, $C_{p}=C_{v}+1$ is the specific heat at constant pressure and the Prandtl number is $\operatorname{Pr}=C_{p} \mu / \kappa ; \gamma=C_{p} / C_{v}$ is the adiabatic exponent which can be freely adjusted.

Using the concept of shifted lattices [23], the discrete velocities $\boldsymbol{v}_{i}$ are written in a reference frame moving with a constant velocity $\boldsymbol{U}$

$$
\boldsymbol{v}_{i}=\boldsymbol{c}_{i}+\boldsymbol{U}
$$

Here, we use $\boldsymbol{U}=(U, 0)$ (moving reference frame in the $x$ direction). In this way, deviations in the pertinent higher-order moments are minimized whenever the flow velocity is around
$\boldsymbol{U}$ [see Eq. (25) below] and that in turn can increase the operating range of the model in terms of flow velocity. Further details on shifted lattices can be found in Ref. [23].

For standard set of discrete velocities, $Q=9$ in $D=2$,

$$
\boldsymbol{c}_{i}=\left(c_{i x}, c_{i y}\right), \quad i=0, \ldots, \quad Q-1 ; \quad c_{i \alpha} \in\{-1,0,+1\},
$$

the equilibrium $f$ populations can be in a product form as

$$
f_{i}^{\mathrm{eq}}=\rho \Phi_{c_{i x}} \Phi_{c_{i y}},
$$

where

$$
\begin{gathered}
\Phi_{-1}=\frac{-\left(u_{\alpha}-U_{\alpha}\right)+\left(u_{\alpha}-U_{\alpha}\right)^{2}+T}{2}, \\
\Phi_{0}=1-\left[\left(u_{\alpha}-U_{\alpha}\right)^{2}+T\right] \\
\Phi_{+1}=\frac{\left(u_{\alpha}-U_{\alpha}\right)+\left(u_{\alpha}-U_{\alpha}\right)^{2}+T}{2},
\end{gathered}
$$

and $\alpha=x, y$.

The populations $g_{i}^{\mathrm{eq}}, g_{i}^{*}$ are constructed using the following general form:

$$
\begin{aligned}
G_{(0,0)}= & M_{0}\left(1-U^{2}-T+2 T^{2}\right)+M_{x} 2 U(1-T) \\
& -M_{x x} \frac{1}{2}(1+T)+M_{y y} \frac{1}{2}\left(2 U^{2}-1-T\right),
\end{aligned}
$$

$$
\begin{aligned}
G_{(\sigma, 0)}= & M_{0}\left(\frac{U^{2}}{2}-\sigma \frac{U}{2}+\frac{1}{2} T-T^{2}\right)-M_{x}\left(U-\frac{\sigma}{2}\right)(1-T) \\
& +M_{x x} \frac{1}{4}(1+T)+M_{y y} \frac{1}{4}\left(-2 U^{2}+2 \sigma U+T-1\right),
\end{aligned}
$$

$$
\begin{aligned}
G_{(0, \lambda)}= & M_{0}\left(\frac{T}{2}-T^{2}\right)+M_{y} \lambda\left(\frac{1}{2}-\frac{U^{2}}{2}-\frac{T}{2}\right)+M_{x} U T \\
& -M_{x x} \frac{1}{4}(1-T)+M_{x y} \lambda(U) \\
+ & M_{y y} \frac{1}{2}\left(\frac{1}{2}-U^{2}+\frac{T}{2}\right) \\
G_{(\sigma, \lambda)}= & M_{0}\left(-\frac{T}{4}+\frac{T^{2}}{2}\right)+M_{x} T\left(-\frac{U}{2}+\sigma \frac{1}{4}\right) \\
& +M_{y}\left[\frac{\lambda}{4}\left(U^{2}+T-\sigma U\right)\right]+M_{x x} \frac{1}{8}(1-T) \\
& +M_{x y}\left(\sigma \lambda \frac{1}{4}-\lambda \frac{U}{2}\right) \\
& +M_{y y} \frac{1}{2}\left(\frac{U^{2}}{2}-\sigma \frac{U}{2}+\frac{1-T}{4}\right)
\end{aligned}
$$

where $\sigma, \lambda=\{+1,-1\}$ and the two indices are identified with the components of the discrete velocity vectors $G_{\left(c_{i x}, c_{i y}\right)}$ and, thus, enumerate all nine populations. The moments required 
TABLE I. Moments needed for the computation of $g_{i}^{\mathrm{eq}}$ and $g_{i}^{*}$ [see Eq. (9) for the nomenclature of discrete speeds].

\begin{tabular}{lccc}
\hline \hline$G_{i}$ & $M_{0}$ & $M_{\alpha}$ & $M_{\alpha \beta}$ \\
\hline$g_{i}^{\text {eq }}$ & $2 \rho E$ & $q_{\alpha}^{\text {eq }}$ & $R_{\alpha \beta}^{\text {eq }}$ \\
$g_{i}^{*}$ & $2 \rho E$ & $q_{\alpha}^{*}$ & $R_{\alpha \beta}^{\text {eq }}$ \\
\hline \hline
\end{tabular}

for the computations are provided in Table I and defined as

$$
\begin{gathered}
q_{\alpha}^{\mathrm{eq}}=\sum_{i=0}^{Q-1} v_{i \alpha} g_{i}^{\mathrm{eq}}=2 \rho u_{\alpha}(E+T), \\
R_{\alpha \beta}^{\mathrm{eq}}=\sum_{i=0}^{Q-1} v_{i \alpha} v_{i \beta} g_{i}^{\mathrm{eq}}=2 \rho E\left(T \delta_{\alpha \beta}+u_{\alpha} u_{\beta}\right) \\
\quad+2 \rho T\left(T \delta_{\alpha \beta}+2 u_{\alpha} u_{\beta}\right), \\
q_{\alpha}^{*}=\sum_{i=0}^{Q-1} v_{i \alpha} g_{i}^{*}=q_{\alpha}^{\mathrm{eq}}+2 u_{\beta}\left(P_{\alpha \beta}-P_{\alpha \beta}^{\mathrm{eq}}\right), \\
P_{\alpha \beta}^{\mathrm{eq}}=\sum_{i=0}^{Q-1} v_{i \alpha} v_{i \beta} f_{i}^{\mathrm{eq}}=\rho u_{\alpha} u_{\beta}+\rho T \delta_{\alpha \beta} .
\end{gathered}
$$

Finally, the correction terms $\phi_{i}$ can be computed as [22]

$$
\phi_{i}=A_{i \alpha} X_{\alpha},
$$

where

$$
\begin{gathered}
X_{\alpha}=-\partial_{\beta}\left[\left(\frac{\mu}{\rho T}\right) \partial_{\gamma} Q_{\alpha \beta \gamma}^{\prime}\right], \\
A_{i \alpha}=c_{i \alpha}-\frac{1}{2} c_{i \alpha} c_{i}^{2},
\end{gathered}
$$

and $Q_{\alpha \beta \gamma}^{\prime}$ is the deviation term in the third-order equilibrium moment,

$Q_{\alpha \beta \gamma}^{\prime}=\left\{\begin{array}{c}\rho\left(u_{\alpha}-U_{\alpha}\right)(1-3 T)-\rho\left(u_{\alpha}-U_{\alpha}\right)^{3}, \text { if } \alpha=\beta=\gamma, \\ 0, \text { if } \alpha \neq \beta, \text { or } \alpha \neq \gamma, \text { or } \beta \neq \gamma .\end{array}\right.$

In general, large magnitude of error terms (23) may result in numerical instability and therefore, it is necessary to employ appropriate shifted velocity $\boldsymbol{U}$, for the simulation of supersonic flows. Thus, the presence of flow dependent correction terms limits the robustness of the present model. Finally, we note that while the two-dimensional D2Q9 lattice is used for the sake of presentation, all the above can be applied to the three-dimensional lattice D3Q27.

\section{Hydrodynamic limit}

Using the Chapman-Enskog analysis, it can be shown that the kinetic equations (1) and (2) recover the full NSF equations in the hydrodynamic limit [22],

$$
\begin{gathered}
\partial_{t} \rho+\partial_{\alpha}\left(\rho u_{\alpha}\right)=0, \\
\rho \partial_{t} u_{\alpha}+\rho u_{\beta} \partial_{\beta} u_{\alpha}=-\partial_{\alpha} p-\partial_{\beta} \Pi_{\alpha \beta}, \\
\rho C_{v} \partial_{t} T+\rho u_{\alpha} \partial_{\alpha} T=-p \partial_{\alpha} u_{\alpha}-\Pi_{\alpha \beta} \partial_{\alpha} u_{\beta} \\
-\partial_{\alpha}\left(-\kappa \partial_{\alpha} T\right),
\end{gathered}
$$

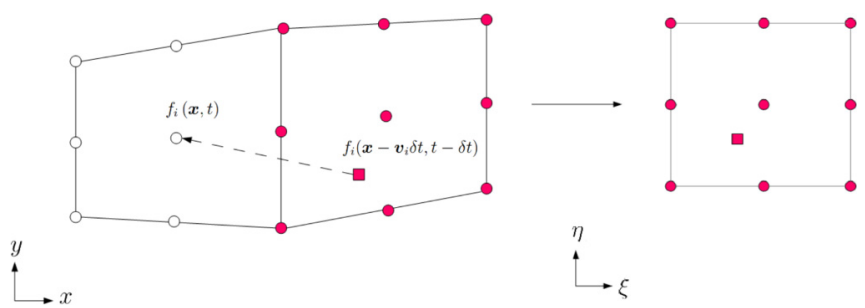

FIG. 1. Schematic of a second-order finite-element mesh, the semi-Lagrangian propagation along the discrete velocity $\boldsymbol{v}_{i}$ and mapping from the global coordinate $(x, y)$ to local coordinate $(\xi, \eta)$.

where $p=\rho T$ is the pressure, $D$ is the dimension, and $\Pi_{\alpha \beta}$ is the viscous stress tensor defined as

$$
\Pi_{\alpha \beta}=-\mu\left[S_{\alpha \beta}-\frac{2}{D} \partial_{\gamma} u_{\gamma} \delta_{\alpha \beta}\right]+\zeta \partial_{\gamma} u_{\gamma} \delta_{\alpha \beta},
$$

with the strain rate tensor,

$$
S_{\alpha \beta}=\partial_{\alpha} u_{\beta}+\partial_{\beta} u_{\alpha},
$$

and the bulk viscosity given by

$$
\zeta=\left(\frac{1}{C_{v}}-\frac{2}{D}\right) \mu
$$

We shall now proceed with the numerical implementation of the model.

\section{NUMERICAL IMPLEMENTATION}

\section{A. Semi-Lagrangian propagation on an unstructured mesh}

The semi-Lagrangian propagation is a practical generalization of standard LB propagation, which removes the restriction related to the regular lattice by performing interpolation in order to find the solution at the departure points [25]. Here we employ the second-order finite-element interpolation scheme to reconstruct solution at the departure points. An example of a semi-Lagrangian propagation on a second-order finite-element mesh with nine collocation points is presented in Fig. 1. It has been shown that this type of reconstruction is less dissipative compared to other off-lattice schemes [25] and also it has been applied successfully to LB for simulation of incompressible turbulent flows [26].

The semi-Lagrangian propagation at the departure point of characteristic lines $\boldsymbol{x}-\boldsymbol{v}_{i} \delta t$ is then written as

$$
f_{i}(\boldsymbol{x}, t)=f_{i}\left(\boldsymbol{x}-\boldsymbol{v}_{i} \delta t, t-\delta t\right)=\sum_{s=1}^{9} f_{i}\left(\boldsymbol{\xi}_{s}, t-\delta t\right) N_{s}\left(\boldsymbol{\xi}_{\mathrm{dp}}\right),
$$

where $N_{s}\left(\boldsymbol{\xi}_{\mathrm{dp}}\right)$ denotes the values of the shape functions, written in the local coordinate system $\xi=(\xi, \eta),(-1 \leqslant \xi, \eta \leqslant$ 1), at the departure point (red square in Fig. 1), and $s=9$ is number of collocation points. Here the second-order quadratic shape functions are used as follows [27]:

$$
\begin{array}{ll}
N_{1}=\frac{1}{4} \xi \eta(\xi-1)(\eta-1), & N_{2}=\frac{1}{4} \xi \eta(\xi+1)(\eta-1), \\
N_{3}=\frac{1}{4} \xi \eta(\xi+1)(\eta+1), & N_{4}=\frac{1}{4} \xi \eta(\xi-1)(\eta+1), \\
N_{5}=\frac{1}{2} \eta\left(1-\xi^{2}\right)(\eta-1), & N_{6}=\frac{1}{2} \xi(\xi+1)\left(1-\eta^{2}\right),
\end{array}
$$




$$
\begin{gathered}
N_{7}=\frac{1}{2} \eta\left(1-\xi^{2}\right)(\eta+1), \quad N_{8}=\frac{1}{2} \xi(\xi-1)\left(1-\eta^{2}\right), \\
N_{9}=\left(1-\xi^{2}\right)\left(1-\eta^{2}\right) .
\end{gathered}
$$

Therefore, semi-Lagrangian propagation on unstructured finite-element mesh requires two steps: First, computing the local coordinates of the departure point $\xi_{\mathrm{dp}}$ (see Fig. 1) which, for quadrilateral elements, involves solving a nonlinear system of equations resulting from

$$
\boldsymbol{x}_{\mathrm{dp}}=\sum_{s=1}^{4} \boldsymbol{x}_{s} N_{s}\left(\boldsymbol{\xi}_{\mathrm{dp}}\right),
$$

where in order to simplify the computation, four vertices are used to define shape functions,

$$
\begin{array}{ll}
N_{1}=\frac{1}{4}(1-\xi)(1-\eta), & N_{2}=\frac{1}{4}(1+\xi)(1-\eta), \\
N_{3}=\frac{1}{4}(1+\xi)(1+\eta), & N_{4}=\frac{1}{4}(1-\xi)(1+\eta) .
\end{array}
$$

Second, the values of the populations at the departure point are computed by means of the values of the populations at collocation points (red circles) using Eq. (32).

After the propagation, the correction terms $\phi_{i}$ are obtained using (22). However, the computation of correction terms requires the knowledge of spatial gradients for deviation terms (25). This is done using the finite-element formula for the first-order derivative. For a generic variable $Q$, we can write

$$
\partial_{x} Q=\boldsymbol{J}^{-1} \sum_{s} Q_{s} \partial_{\xi} N_{s},
$$

where $Q_{s}$ are the values of $Q$ at collocation points and $J^{-1}$ is the inverse of the Jacobian matrix of transformation computed with

$$
\boldsymbol{J}^{-1}=\frac{1}{\operatorname{det} \boldsymbol{J}}\left[\begin{array}{cc}
\partial_{\eta} y & -\partial_{\xi} y \\
-\partial_{\eta} x & \partial_{\xi} x
\end{array}\right]
$$

and

$$
\operatorname{det} \boldsymbol{J}=\partial_{\xi} x \partial_{\eta} y-\partial_{\xi} y \partial_{\eta} x
$$

is the determinant of the Jacobian matrix. The metrics of transformation $\partial_{\xi} x, \partial_{\eta} x, \partial_{\xi} y, \partial_{\eta} y$ are computed with the following formula:

$$
\partial_{\xi} \boldsymbol{x}=\sum_{s} \boldsymbol{x}_{s} \partial_{\xi} N_{s}
$$

Note that the nodes on the element edges are assigned to the element with the larger area.

Finally, the postcollision populations are computed in the same way as in the standard LB method.

\section{B. Wall boundary conditions}

Semi-Lagrangian propagation on unstructured grid makes it possible to employ body-fitted mesh and simulate complex geometries. Therefore, an appropriate wall boundary condition (BC) is required. Here we follow the approach proposed by Ref. $[28,29]$ and replace the missing populations during propagation, with the following expression:

$$
\begin{aligned}
f_{i}^{\mathrm{miss}}= & f_{i}^{\mathrm{eq}}\left(\rho_{\mathrm{tgt}}, \boldsymbol{u}_{\mathrm{tgt}}, T_{\mathrm{tgt}}\right) \\
& +\delta t f_{i}^{(1)}\left(\rho_{\mathrm{tgt}}, \boldsymbol{u}_{\mathrm{tgt}}, T_{\mathrm{tgt}}, \nabla \boldsymbol{u}_{\mathrm{tgt}}, \nabla T_{\mathrm{tgt}}\right),
\end{aligned}
$$

TABLE II. Moments needed for the computation of $f_{i}^{(1)}$ and $g_{i}^{(1)}$.

\begin{tabular}{lccc}
\hline \hline$G_{i}$ & $M_{0}$ & $M_{\alpha}$ & $M_{\alpha \beta}$ \\
\hline$f_{i}^{(1)}$ & 0 & 0 & $P_{\alpha \beta}^{(1)}$ \\
$g_{i}^{(1)}$ & 0 & $q_{\alpha}^{(1)}$ & $R_{\alpha \beta}^{(1)}$ \\
\hline \hline
\end{tabular}

$$
\begin{aligned}
g_{i}^{\mathrm{miss}}= & g_{i}^{\mathrm{eq}}\left(\rho_{\mathrm{tgt}}, \boldsymbol{u}_{\mathrm{tgt}}, T_{\mathrm{tgt}}\right) \\
& +\delta t g_{i}^{(1)}\left(\rho_{\mathrm{tgt}}, \boldsymbol{u}_{\mathrm{tg}}, T_{\mathrm{tgt}}, \nabla \boldsymbol{u}_{\mathrm{tgt}}, \nabla T_{\mathrm{tgt}}\right),
\end{aligned}
$$

where $f_{i}^{\mathrm{eq}}, g_{i}^{\mathrm{eq}}$ are equilibrium parts computed from (10) and (14) to (17); $f_{i}^{(1)}, g_{i}^{(1)}$ are nonequilibrium parts; and $\rho_{\mathrm{tgt}}$, $\boldsymbol{u}_{\mathrm{tgt}}$, and $T_{\mathrm{tgt}}$ are target values which need to be specified. The nonequilibrium parts are obtained based on the Grad's approximation and using the general formula (14) to (17) with the nonequilibrium moments given in Table II $[14,30]$

$$
\begin{gathered}
P_{\alpha \beta}^{(1)}=-\frac{1}{\omega} \rho T\left(S_{\alpha \beta}-\frac{1}{C_{v}} \partial_{\gamma} u_{\gamma} \delta_{\alpha \beta}\right), \\
q_{\alpha}^{(1)}=-\frac{2}{\omega_{1}} \rho C_{p} T \partial_{\alpha} T+2 u_{\beta} P_{\alpha \beta}^{(1)}, \\
R_{\alpha \beta}^{(1)}=-\frac{2}{\omega_{1}} \rho T\left[S_{\alpha \beta}(E+2 T)+u_{\alpha} \partial_{\beta} E+u_{\beta} \partial_{\alpha} E\right],
\end{gathered}
$$

where $S_{\alpha \beta}$ is the strain rate tensor.

For computing target values, if missing populations belong to points on the wall (black circles in Fig. 2), target velocities are zero, $\boldsymbol{u}_{\mathrm{tgt}}=\mathbf{0}$ and target density and temperature (for adiabatic wall) are obtained by setting

$$
\begin{aligned}
& \left.\frac{\partial \rho}{\partial \boldsymbol{n}}\right|_{\text {wall }}=0, \\
& \left.\frac{\partial T}{\partial \boldsymbol{n}}\right|_{\text {wall }}=0,
\end{aligned}
$$

where $\boldsymbol{n}$ is the normal direction to the wall boundary $\partial \Omega$. Given the normal direction $\boldsymbol{n}$, its end point $B$ and considering the distance from $A$ to $B$ as $\|\boldsymbol{n}\|=\delta t$, the values of density and temperature at $B$ can be evaluated using a finite-element interpolation. For example for the density, we can write

$$
\rho_{B}=\sum_{s=1}^{9} \rho_{s} N_{s},
$$

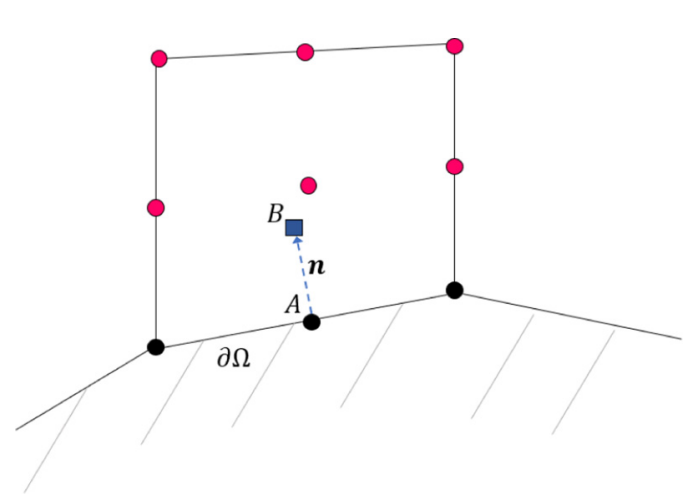

FIG. 2. Schematic representation of the wall boundary condition implementation. 
TABLE III. Accuracy test for the propagation of density perturbation.

\begin{tabular}{lcc}
\hline \hline Element size $\left(\Delta x / L_{x}\right)$ & $L_{\infty}$ error & Order \\
\hline 0.1 & 0.033958 & - \\
0.05 & 0.010144 & 1.75 \\
0.025 & 0.002953 & 1.78 \\
0.0125 & 0.000841 & 1.82 \\
\hline \hline
\end{tabular}

where $N_{s}$ are shape functions and $\rho_{s}$ are the magnitude of density at nine collocation points (circles in Fig. 2). Once $\rho_{B}$ is found, the first-order approximation for the normal derivative is assumed,

$$
\left.\frac{\partial \rho}{\partial \boldsymbol{n}}\right|_{\text {wall }}=\frac{\rho_{B}-\rho_{A}}{\|\boldsymbol{n}\|}=0 .
$$

Therefore, the target value can be approximated as

$$
\rho_{\text {tgt }}=\rho_{A}=\rho_{B} .
$$

It is important to note that if missing populations belong to points which do not lie on the wall boundaries (red circles in Fig. 2), the local quantities of the previous time step are used as target values.

The evaluation of spatial gradients in nonequilibrium moments is performed using (41). It was demonstrated in Ref. [29] that the first-order accurate evaluation of spatial derivatives is sufficient.

\section{RESULTS}

In this section, the model presented above is validated numerically through simulation of four benchmark cases. All simulations are performed with $\gamma=1.4, \operatorname{Pr}=0.71$, the D2Q9 lattice model, and adiabatic wall assumption. The time step used in this study is $\delta t=\delta x_{\min } / 1.5$, which corresponds to the $\mathrm{CFL}=\frac{\max \left|v_{i}\right| \delta t}{\delta x_{\min }}=0.66$, where $\delta x_{\min }$ is the minimum spacing between any two points of the computational mesh.

\section{A. Accuracy test}

The smooth density propagation [31] is solved in order to test the accuracy of the present model on unstructured mesh. The initial condition of the flow field is given by

$$
\begin{aligned}
\rho & =\rho_{\text {ref }}+0.2 \sin \left(2 \pi x / L_{x}\right) \sin \left(2 \pi y / L_{y}\right), \\
u & =\operatorname{Ma} \sqrt{\gamma T_{\text {ref }}}, \\
v & =0, \\
T & =\rho_{\text {ref }} T_{\text {ref }} / \rho,
\end{aligned}
$$

with the domain size $L_{x}=L_{y}=8000$, reference density $\rho_{\text {ref }}=1$, reference temperature $T_{\text {ref }}=0.2$, and $\mathrm{Ma}=0.2$. We compute the solution after two periods of propagation in order to evaluate the convergence order of the scheme using four different uniform grids and based on the $L_{\infty}$ error of density. As shown in Table III, the accuracy in space is slightly below second order. This is consistent with previous results on semi-Lagrangian LB for incompressible flows as reported in Refs. [25,26].
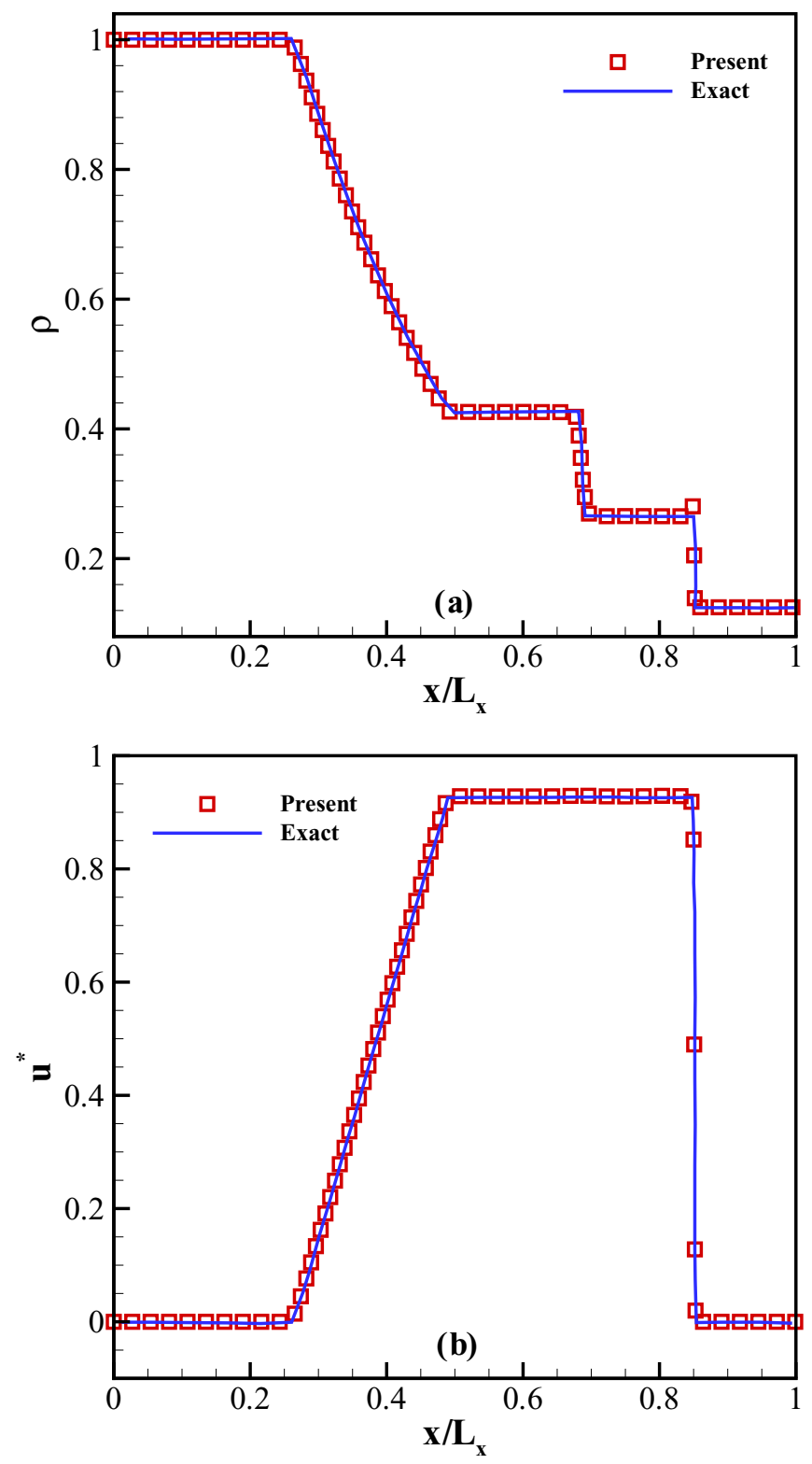

FIG. 3. Sod shock tube simulation results at nondimensional time $t^{*}=0.2$ : (a) density and (b) reduced velocity. Symbols: present model; line: exact solution.

\section{B. Sod shock tube}

The Sod shock tube problem [32] is a classical Riemann problem to test the capability of the model when shock and expansion waves are present in the flow field. The initial flow field for this problem is given by

$$
\left(\rho, u_{x}, u_{y}, p\right)=\left\{\begin{array}{l}
(1.0,0,0,0.2), \quad x / L_{x} \leqslant 0.5, \\
(0.125,0,0,0.02), \quad x / L_{x}>0.5,
\end{array}\right.
$$

where $L_{x}=20$ is the domain length. A uniform grid with $400 \times 5$ elements is used with the viscosity $\mu=0.0001$. Simulation results for the density and reduced velocity $u^{*}=$ $u / \sqrt{T_{L}}\left(T_{L}\right.$ is temperature on the left side of tube) at nondimensional time $t^{*}=t \sqrt{T_{L}} / L_{x}=0.2$, are shown in Fig. 3. It is observed that, apart from small oscillations, the results are in excellent agreement with the exact solutions. 

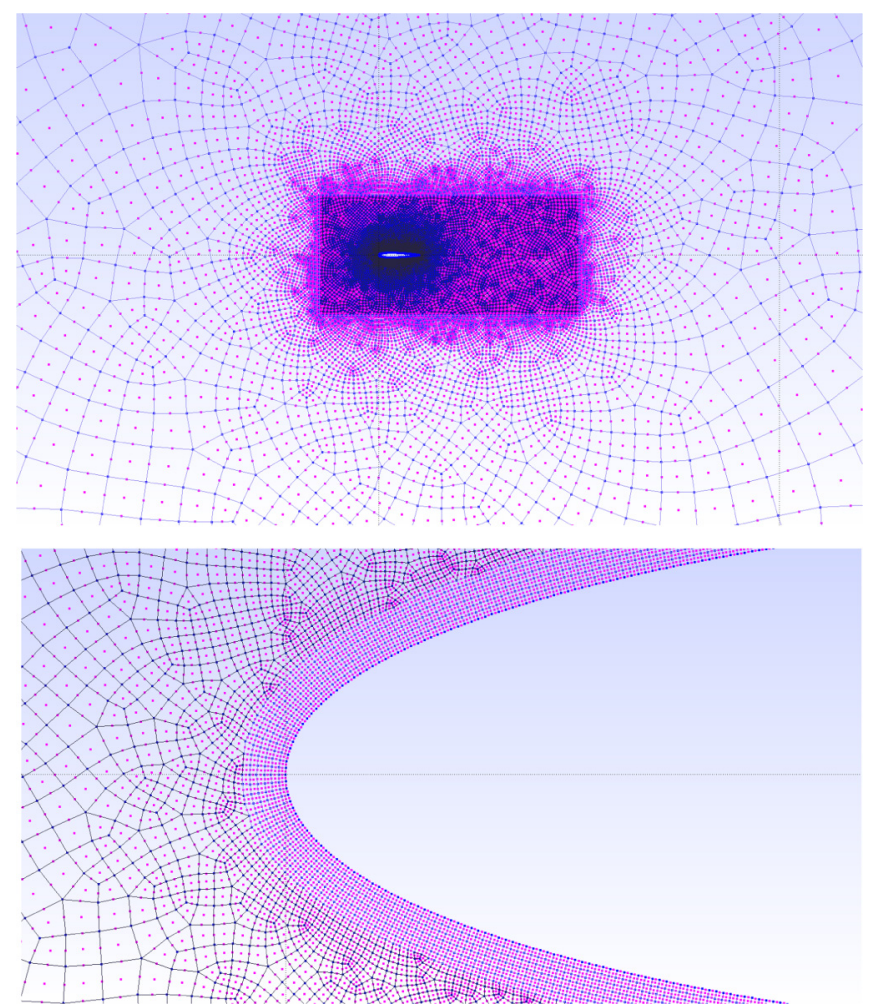

FIG. 4. Second-order finite-element mesh (Mesh-1) used for the simulation of subsonic flow over NACA0012 airfoil. Bottom is the zoom near leading edge of the airfoil.

\section{Subsonic flow over NACA0012 airfoil}

This test case involves a subsonic flow over a NACA0012 airfoil at a Mach number $\mathrm{Ma}=u_{\infty} / \sqrt{\gamma T_{\infty}}=0.5$, incoming temperature $T_{\infty}=0.2$, an angle of attack of $\alpha=0^{\circ}$, and a Reynolds number of $\operatorname{Re}=\rho_{\infty} u_{\infty} c / \mu=5000$ based on the chord length of the airfoil $c$. The simulation is performed on an unstructured quadrilateral mesh with 43235 elements as shown in Fig. 4 (Mesh-1), where a layer of orthogonal grid is used near the airfoil surface and anisotropic grid is used elsewhere. The minimum size of the elements near the airfoil surface $\delta / c \approx 0.0015$. In this case, the flow separation occurs near the tailing edge, which causes the formation of two small re circulation bubbles in the wake, as shown by the Mach number contours in Fig. 5. Pressure coefficient $c_{p}=\left(p-p_{\infty}\right) /\left(0.5 \rho_{\infty} u_{\infty}^{2}\right)$ and skin friction coefficient $c_{f}=$ $\tau_{w} /\left(0.5 \rho_{\infty} u_{\infty}^{2}\right)$, where $\tau_{w}$ is the local wall shear stress, on the airfoil surface are compared in Fig. 6 with the discontinuous Galerkin (DG) solution of the compressible NS equations [33]. Moreover, the comparison of the drag coefficient with other numerical results is shown in Table IV. It can be seen that the present results are in good agreement with all reference data.

To further validate the solver, the numerical results computed at angle of attack $\alpha=2^{\circ}$ are also compared in Fig. 7 with the reference solution reported in Ref. [34]. Note that in this case the flow becomes unsteady in the wake.

Moreover, in order to investigate the effect of grid quality on the solution, we repeat the simulation with another mesh, but with irregular elements close to the airfoil surface, with
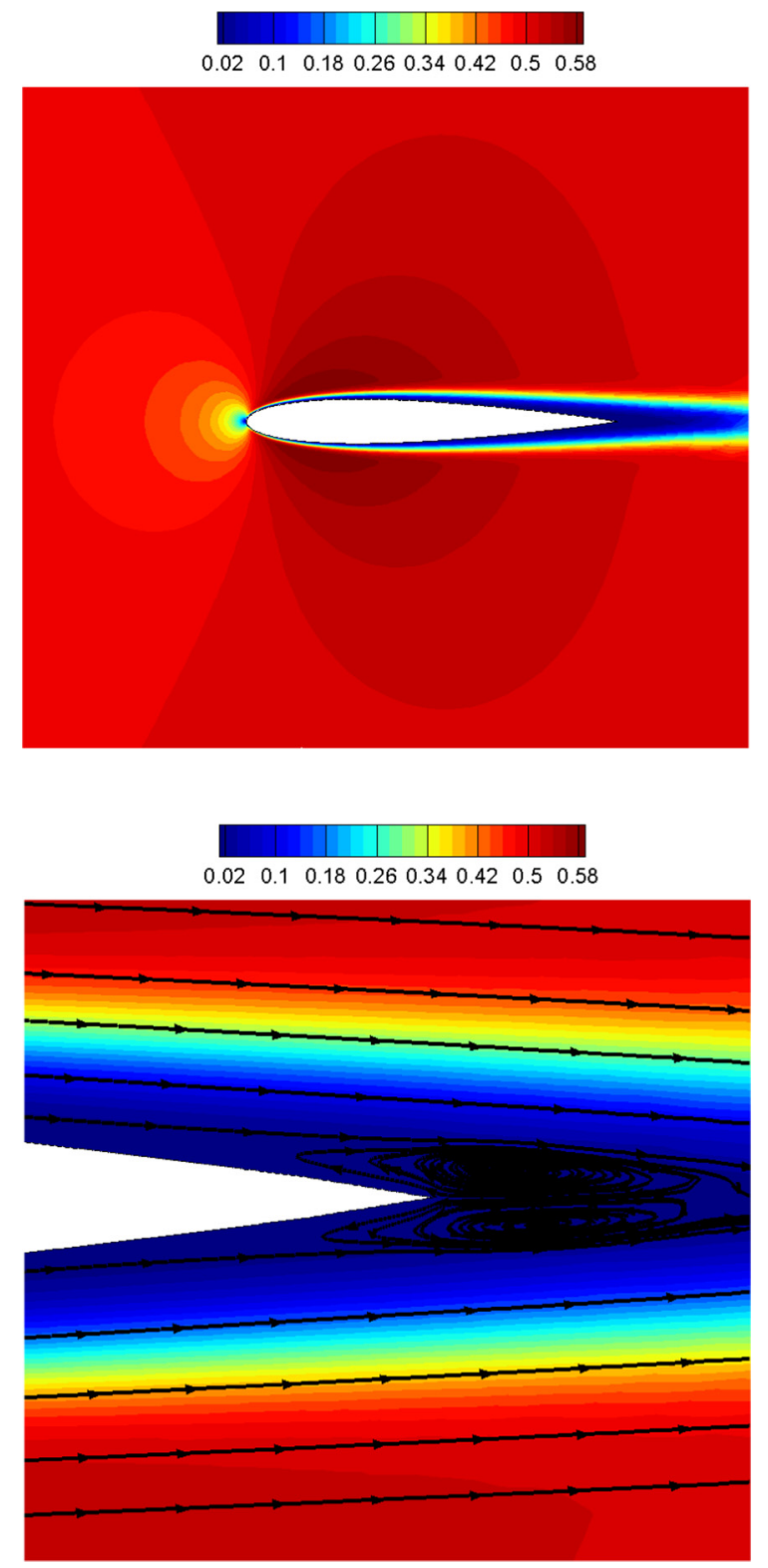

FIG. 5. Mach contour for subsonic flow over NACA0012 airfoil at $\mathrm{Ma}=0.5, \mathrm{Re}=5000$, and $\alpha=0^{\circ}$. Bottom figure shows streamlines near trailing edge.

the same element size near the wall $(\delta / c \approx 0.0015)$, as shown in Fig. 8 (Mesh-2). The results obtained are shown in Fig. 7 in comparison with the results of Mesh- 1 and the reference solution [34]. It is observed that, the results are almost identical. We can therefore conclude that in this case, the mesh quality does not have significant effect on the results. However, the effect of grid quality needs to be further investigated in problems with higher Reynolds number, as it might be necessary to employ a high-quality orthogonal grid near the surface in order to correctly capture the boundary layer.

\section{Unsteady supersonic flow over NACA0012 airfoil}

In order to test the capability of the present model in capturing shock waves on unstructured mesh, the Mach number of the previous setup was increased to $\mathrm{Ma}=1.5$ and the 

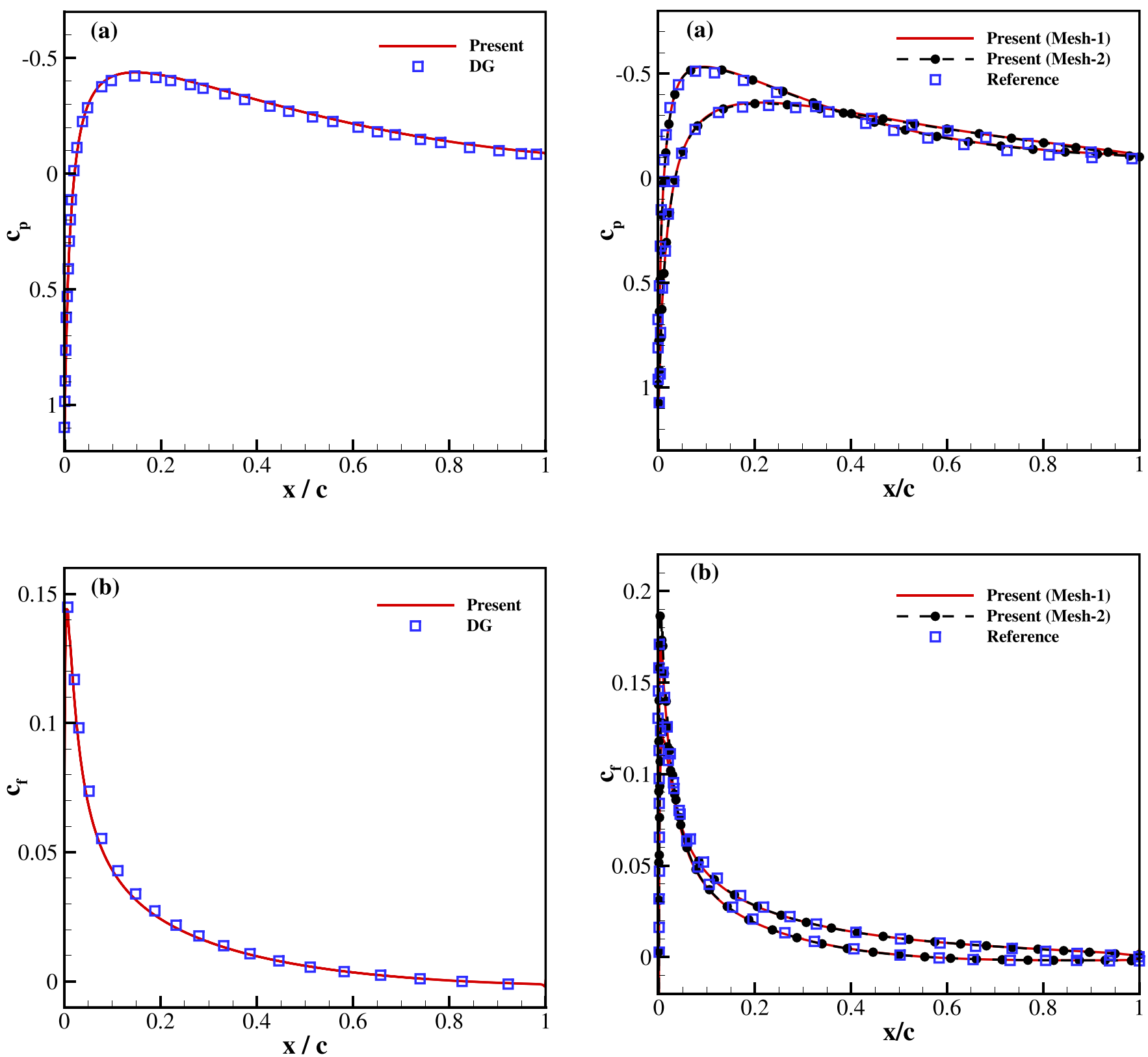

FIG. 6. Distribution of (a) pressure coefficient and (b) skin friction coefficient on the NACA0012 airfoil surface for subsonic flow at $\mathrm{Ma}=0.5, \operatorname{Re}=5000$, and $\alpha=0^{\circ}$. Line: present model; symbols: DG solver [33].

Reynolds number was set to $\operatorname{Re}=10000$. As flow is supersonic, shifted lattice with $U_{x}=0.3$ was used and unstructured mesh with 143123 quadrilateral elements and minimum element size of $\delta / c=0.0015$ was employed.

TABLE IV. Drag coefficient for subsonic flow over NACA0012 airfoil at $\mathrm{Ma}=0.5, \operatorname{Re}=5000$, and $\alpha=0^{\circ}$.

\begin{tabular}{lc}
\hline \hline Method & $c_{d}$ \\
\hline Direct DG [33] & 0.05543 \\
Reconstructed DG [35] & 0.05534 \\
Spectral difference [36] & 0.05476 \\
Present & 0.05568 \\
\hline \hline
\end{tabular}

FIG. 7. Distribution of (a) pressure coefficient and (b) skin friction coefficient on the NACA0012 airfoil surface for subsonic flow at $\mathrm{Ma}=0.5, \operatorname{Re}=5000$, and $\alpha=2^{\circ}$. Lines: present model; symbols: reference solution [34].

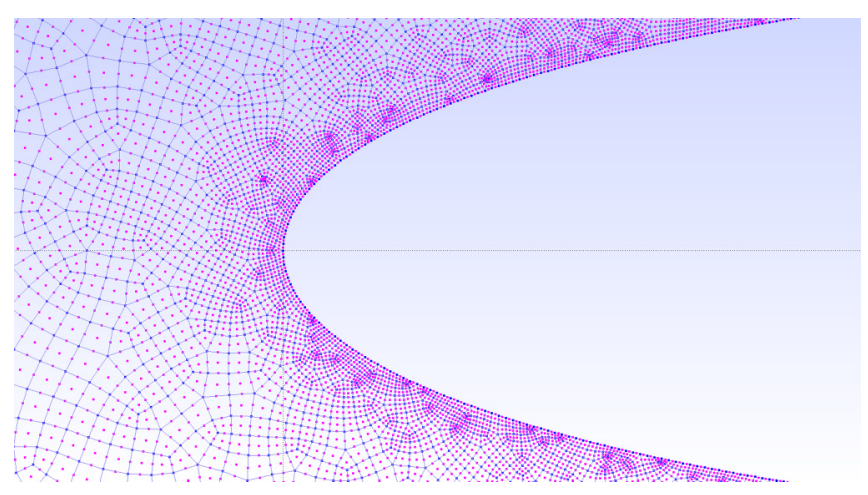

FIG. 8. Mesh-2 used for the simulation of subsonic flow over NACA0012 airfoil. 

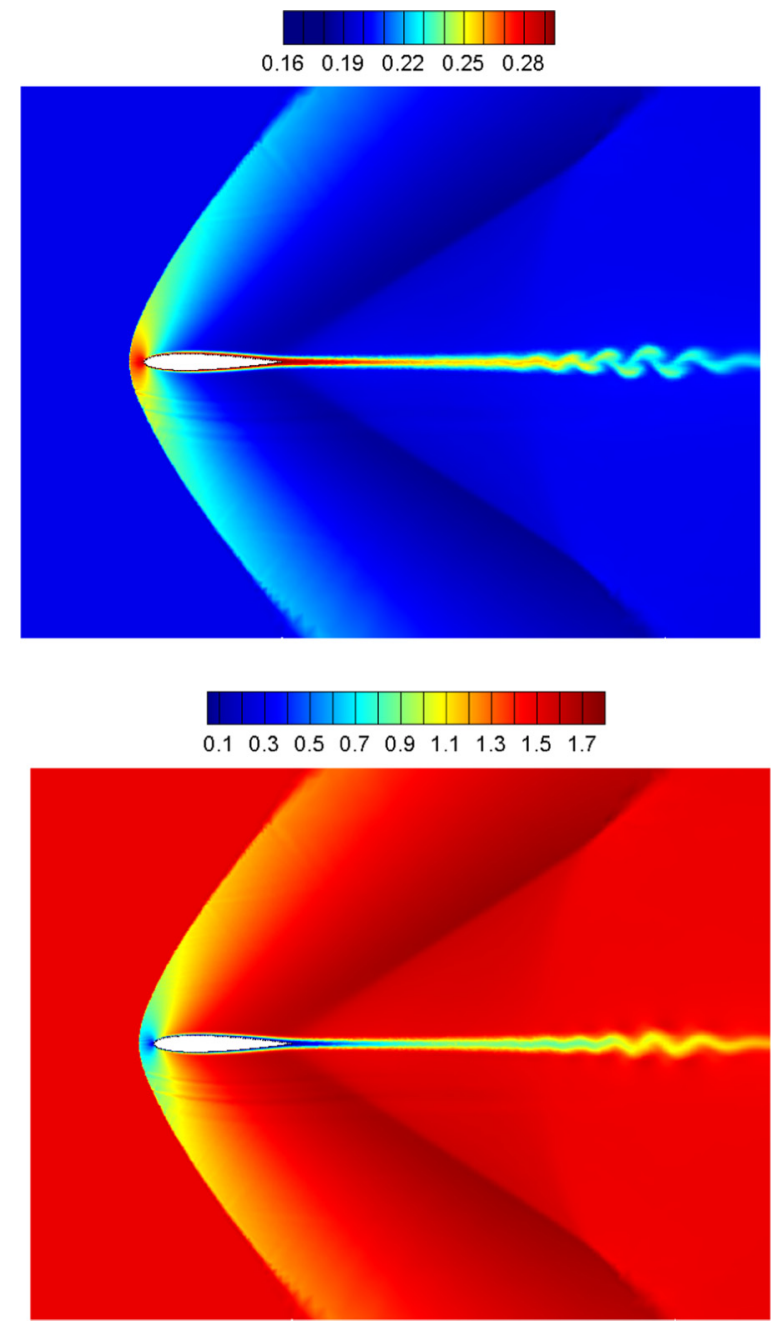

FIG. 9. Temperature (top) and Mach number (bottom) contours for supersonic flow over NACA0012 airfoil at $\mathrm{Ma}=1.5, \mathrm{Re}=$ 10000 .

Figure 9 shows the temperature and Mach contours. It is observed that a bow shock is formed in front of the airfoil and oblique shocks appear from the trailing edge. Moreover, vortex shedding is started downstream, due to the shear layer developing from the trailing edge boundary layer of the airfoil. To quantify the results, in Fig. 10 the pressure coefficient upstream, downstream, and on the airfoil surface is compared to the numerical solution reported in Ref. [37] and also with the solution of the entropic LBM (ELBM) with D2Q49 lattice model [39]. Good agreement is observed and the present method captures the pre- and postshock values and the shock location with good accuracy.

\section{E. Shock-vortex interaction in Schardin's problem}

Finally, the so called Schardin's problem $[38,40]$ is considered in which a planar shock wave impinges on a finite wedge is reflected and diffracted. The impingement creates a complex shock-shock and shock-vortex interaction [38]. This test case shows the ability of the scheme in handling complex geometries at high-speed flows. Here a shock Mach number $\mathrm{Ma}_{s}=1.34$ is considered and the Reynolds number based on

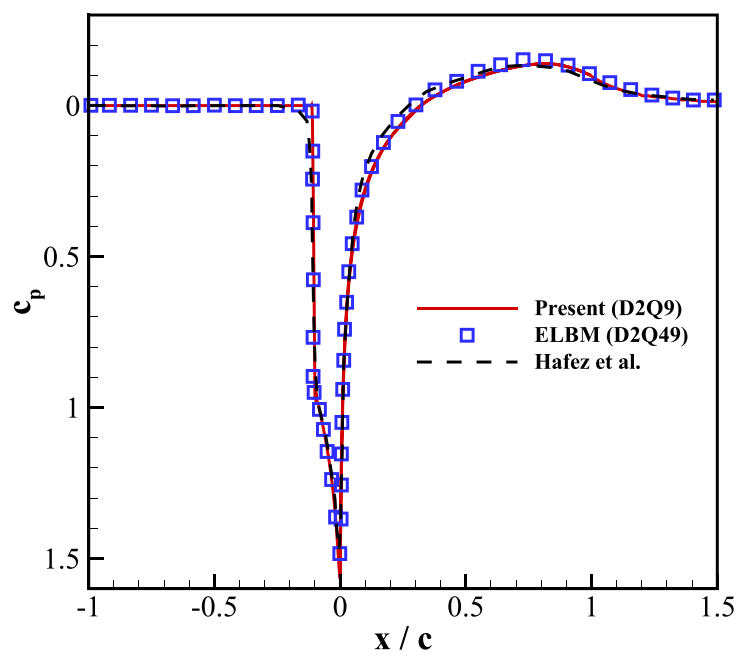

FIG. 10. Pressure coefficient upstream, downstream and on the NACA0012 airfoil surface for supersonic flow at $\mathrm{Ma}=1.5, \mathrm{Re}=$ 10 000. Line: present model with D2Q9 lattice; symbols: ELBM solution with $D 2 Q 49$ lattice; dashed line: results reported in Ref. [37].

wedge length $L$ is set to $\operatorname{Re}=2000$. Further details on this setup can be found in Ref. [38]. Moreover, shifted lattice with $U_{x}=0.3$ is used. Figure 11 shows the evolution of flow field by plotting the density distribution over time. It is observed that the traveling shock wave creates two vortices at the two corners and then interacts with its mirrored counterpart and refracts. Moreover, the time evolution of the position of the triple points $T_{1}$ and $T_{2}$ (shown in Fig. 11), where the reflected and the traveling shocks meet, is compared in Fig. 12 with the experimental results [38] and numerical results of ELBM with D2Q49 lattice model [14]. Once again, the results obtained are in good agreement with those solutions, which shows the accuracy of the present model.

\section{CONCLUSIONS}

We presented an extension of the compressible lattice Boltzmann model on standard lattices [22] for the simulation of compressible flows over complex geometries on unstructured mesh. The extension is based on the semi-Lagrangian propagation on unstructured finite-element mesh and Grad's approximation for replacing missing populations near the wall boundaries. The model was validated by simulating four benchmark test cases, including Sod shock tube, subsonic or supersonic flow over NACA0012 airfoil, and shock-vortex interaction in Schardin's problem. Some remarks about the present study are as follows:

(i) It was shown that the results obtained with the present model on standard lattice are in good agreement with the available numerical and experimental results in the literature.

(ii) The present model with the help of shifted lattices can successfully capture moderately supersonic shock waves on anisotropic meshes without using any artificial dissipation or limiters.

(iii) In this study, the simple BGK collision term was used. It is, therefore, not surprising that in cases with strong discontinuity or high Reynolds number, instability arises at $\mathrm{CFL}=1$, i.e., $d t=d x_{\min }$. Therefore, a CFL number of 

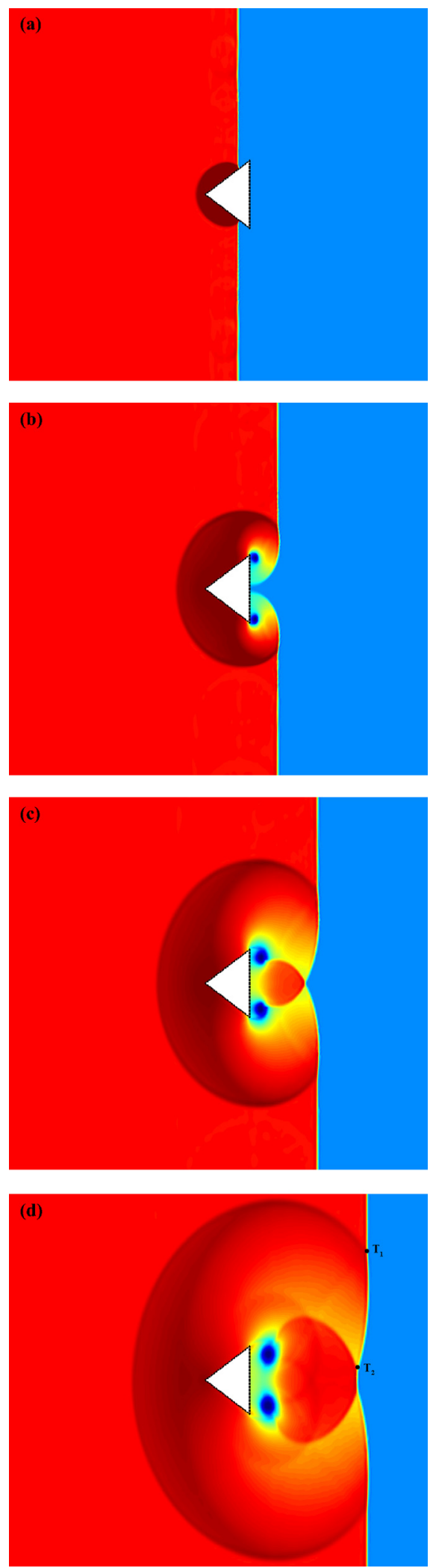

FIG. 11. Evolution of the density for the Schardin's problem at shock Mach number $\mathrm{Ma}_{s}=1.34 . T_{1}$ and $T_{2}$ are triple points.

0.66 was chosen in order to increase the robustness of the scheme. Nevertheless, this CFL number is still considerably higher than those of many other off-lattice schemes (see, e.g.,

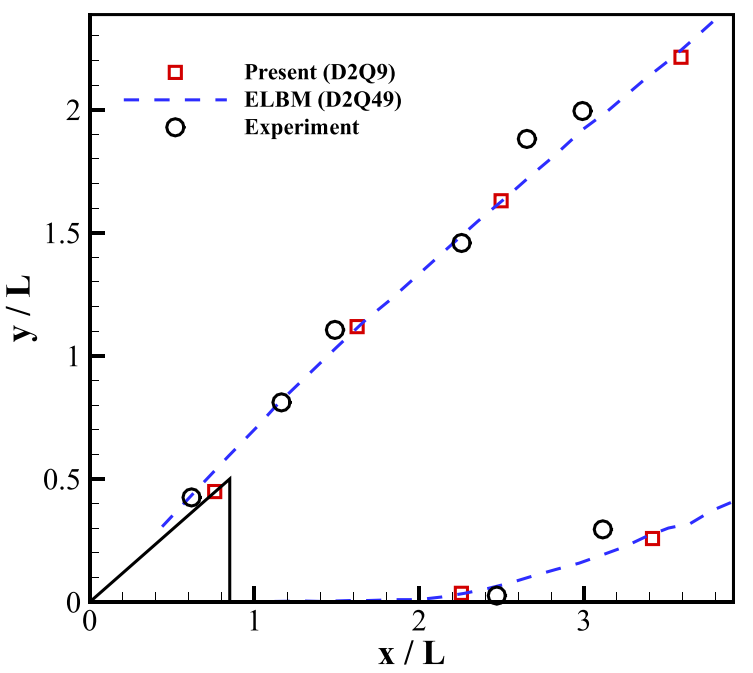

FIG. 12. Comparison of the position of triple points $T_{1}$ and $T_{2}$ for the Schardin's problem. Squares: present model with D2Q9 lattice; dashed line: ELBM solution with $D 2 Q 49$ lattice [14]; circles: experiment [38].

Ref. [24]). Development of more advanced collision terms for increasing the stability of the model is topic for future research.

(iv) Employing a global constant time step based on the minimum spacing in the whole domain is necessary for accurate simulation of unsteady flows. However, it is not efficient in coarse regions of domain and thus is not the best candidate for steady-state simulations. While numerous studies have been done on application of local time stepping within the framework of Eulerian methods, very limited number of works can be found in the literature about adaptive time stepping in semi-Lagrangian scheme in general. Therefore, the effect of using variable time-stepping scheme on the accuracy and stability of the semi-Lagrangian scheme is not generally known and needs to be further investigated. The application of local adaptive time-stepping scheme for accelerating the convergence rate of the steady-state solution will be the focus of our future work.

(v) While the standard LB models with exact streaming on regular space filling lattice have been shown to be highly optimized in terms of computational efficiency, they face with several drawbacks. Standard on-LB models need special treatments such as grid refinement in order to reduce the number of grid points needed in problems involving complex geometries. On the other hand, the computational cost dramatically increases in the case of compressible flows, where a higher-order lattice (like the D2Q25 or D2Q49 lattice models) along with a more sophisticated collision scheme (like the entropic scheme) should be additionally employed for a successful simulation [14]. Therefore, in general, we can say that the computational overhead of performing interpolation in the present model with the D2Q9 lattice model and the BGK collision term is largely, if not fully, compensated by the reduction in computational cost related to a smaller lattice and a simpler collision term. Therefore, due to its relative simplicity and efficiency compared to that of the standard LB 
with larger velocity sets, the present model can be useful to simulate compressible flows involving moderate shock waves on unstructured grids.

(vi) The present model is valid as long as correction terms remain small, which can be achieved by choosing shift velocity frame appropriately.

(vii) An extension to a moving mesh approach would make the present model a suitable candidate for simulation of flows with deformable moving bodies and fluid-solid interaction applications. Extension of the model to three di- mensions is, in principle, straightforward and the subject of future effort.

\section{ACKNOWLEDGMENTS}

This work was supported by the ETH research Grant No. ETH-13 17-1 and the European Research Council (ERC) Advanced Grant No. 834763-PonD. The computational resources at the Swiss National Super Computing Center CSCS were provided under the Grant No. s897. The authors thank Benedikt Dorschner for useful discussions.
[1] F. Higuera, S. Succi, and R. Benzi, Europhys. Lett. 9, 345 (1989).

[2] R. Benzi, S. Succi, and M. Vergassola, Phys. Rep. 222, 145 (1992).

[3] R. Benzi and S. Succi, J. Phys. A 23, L1 (1990).

[4] S. Chikatamarla, C. Frouzakis, I. Karlin, A. Tomboulides, and K. Boulouchos, J. Fluid Mech. 656, 298 (2010).

[5] A. Mazloomi M, S. S. Chikatamarla, and I. V. Karlin, Phys. Rev. Lett. 114, 174502 (2015).

[6] C. Lin, K. H. Luo, L. Fei, and S. Succi, Sci. Rep. 7, 14580 (2017).

[7] X. Shan, X.-F. Yuan, and H. Chen, J. Fluid Mech. 550, 413 (2006).

[8] P. J. Dellar, J. Comput. Phys. 237, 115 (2013).

[9] M. Mendoza, B. M. Boghosian, H. J. Herrmann, and S. Succi, Phys. Rev. Lett. 105, 014502 (2010).

[10] S. Succi, Europhys. Lett. 109, 50001 (2015).

[11] I. Karlin and P. Asinari, Physica A (Amsterdam) 389, 1530 (2010).

[12] Q. Li, Y. L. He, Y. Wang, and W. Q. Tao, Phys. Rev. E 76, 056705 (2007).

[13] N. Frapolli, S. S. Chikatamarla, and I. V. Karlin, Phys. Rev. E 92, 061301(R) (2015).

[14] N. Frapolli, S. S. Chikatamarla, and I. V. Karlin, Phys. Rev. E 93, 063302 (2016).

[15] R.-F. Qiu, C.-X. Zhu, R.-Q. Chen, J.-F. Zhu, and Y.-C. You, Comput. Fluids 166, 24 (2018).

[16] N. Frapolli, Entropic lattice boltzmann models for thermal and compressible flows, Ph.D. thesis, ETH Zurich, 2017.

[17] N. I. Prasianakis and I. V. Karlin, Phys. Rev. E 76, 016702 (2007).

[18] Y. Feng, P. Sagaut, and W. Tao, J. Comput. Phys. 303, 514 (2015).

[19] Y. Feng, P. Sagaut, and W.-Q. Tao, Comput. Fluids 131, 45 (2016).

[20] Y. Feng, P. Boivin, J. Jacob, and P. Sagaut, J. Comput. Phys. 394, 82 (2019).
[21] R. Huang, H. Wu, and N. A. Adams, J. Comput. Phys. 392, 227 (2019).

[22] M. H. Saadat, F. Bösch, and I. V. Karlin, Phys. Rev. E 99, 013306 (2019).

[23] N. Frapolli, S. S. Chikatamarla, and I. V. Karlin, Phys. Rev. Lett. 117, 010604 (2016).

[24] A. Zarghami, S. Ubertini, and S. Succi, Int. J. Numer. Methods Heat Fluid Flow 24, 270 (2014).

[25] A. Krämer, K. Küllmer, D. Reith, W. Joppich, and H. Foysi, Phys. Rev. E 95, 023305 (2017).

[26] G. Di Ilio, B. Dorschner, G. Bella, S. Succi, and I. Karlin, J. Fluid Mech. 849, 35 (2018).

[27] O. C. Zienkiewicz, R. L. Taylor, and J. Z. Zhu, The Finite Element Method: Its Basis and Fundamentals (Elsevier, Amsterdam, 2005).

[28] S. Chikatamarla, S. Ansumali, and I. Karlin, Europhys. Lett. 74, 215 (2006).

[29] B. Dorschner, S. S. Chikatamarla, F. Bösch, and I. V. Karlin, J. Comput. Phys. 295, 340 (2015).

[30] G. Pareschi, N. Frapolli, S. S. Chikatamarla, and I. V. Karlin, Phys. Rev. E 94, 013305 (2016).

[31] X. Ji, F. Zhao, W. Shyy, and K. Xu, J. Comput. Phys. 356, 150 (2018).

[32] G. A. Sod, J. Comput. Phys. 27, 1 (1978).

[33] J. Cheng, X. Yang, T. Liu, and H. Luo, J. Comput. Phys. 327, 484 (2016).

[34] R. Swanson and S. Langer, Comput. Fluids 126, 102 (2016).

[35] X. Yang, J. Cheng, H. Luo, and Q. Zhao, Comput. Fluids 168, 216 (2018).

[36] R. Kannan and Z. Wang, Comput. Fluids 39, 2007 (2010).

[37] M. Hafez and E. Wahba, Comput. Fluids 36, 39 (2007).

[38] S.-M. Chang and K.-S. Chang, Shock Waves 10, 333 (2000).

[39] B. Dorschner, N. Frapolli, S. S. Chikatamarla, and I. V. Karlin, Phys. Rev. E 94, 053311 (2016).

[40] H. Schardin, J. Photogr. Sci. 5, 17 (1957). 